\title{
Watching what's coming near increases tactile sensitivity: an experimental investigation.
}

\author{
Lien Van der Biest ${ }^{\mathrm{a} *}$, Valéry Legrain ${ }^{\mathrm{b}}$, Annick De Paepe ${ }^{\mathrm{a}}$, Geert Crombez ${ }^{\mathrm{a}}$ \\ ${ }^{a}$ Department of Experimental-Clinical and Health Psychology, Ghent University, Henri Dunantlaan 2, 9000 Gent, \\ Belgium. \\ ${ }^{\mathrm{b}}$ Institute of Neuroscience, Université catholique de Louvain, Avenue Mounier 53 bte B1.53.04, 1200 Brussels \\ Belgium. \\ * Corresponding author: Department of Experimental-Clinical and Health Psychology, Ghent University, Henri \\ Dunantlaan 2, 9000 Ghent, Belgium. Tel.: +32 926486 92. Fax: +32 926464 89. E-mail: \\ Livdrbie.VanderBiest@Ugent.be
}

\begin{abstract}
During medical examinations, doctors regularly investigate a patient's somatosensory system by approaching the patient with a medical device (e.g. Von Frey hairs, algometer) or with their hands. It is assumed that the obtained results reflect the true capacities of the somatosensory system. However, evidence from crossmodal spatial research suggests that sensory experiences in one modality (e.g. touch) can be influenced by concurrent information from other modalities (e.g. vision), especially near the body (i.e. in peripersonal space). Hence, we hypothesized that seeing someone approaching your body could alter tactile sensitivity in that body-part. In the In Vivo Approaching Object (IVAO) paradigm, participants detected and localized threshold-level vibrotactile stimuli administered on the left of right hand (= tactile targets). In Experiment 1, this was always preceded by the experimenter approaching the same (congruent trials) or the other (incongruent trials) hand with a pen (= visual cue). In Experiment 2, a condition was added in which a point further away from the hands (also left vs. right) was approached. Response Accuracy was calculated for congruent and incongruent trials (Experiment 1 \& 2) and compared between the close and far condition (Experiment 2). As expected, Response Accuracy was higher in congruent trials compared to incongruent trials, but only near the body. As a result, evidence was found for a crossmodal interaction effect between visual and tactile information in peripersonal space. These results suggest that somatosensory evaluations - both medical or research-based - may be biased by viewing an object approaching the body.
\end{abstract}

Keywords: crossmodal interaction, attention, peripersonal space 


\section{INTRODUCTION}

Imagine undergoing a medical examination, such as pressure algometry. Would your response be affected by seeing the doctor approaching you with the algometer? Health care providers often approach and touch the patient with testing devices such as von Frey hairs, algometers, or with their hands. These tests are often part of daily clinical practice but may also be part of specialized sensory evaluation such as the Quantitative Sensory Testing (QST) in patients with neuropathic pain. When these patients are approached and tested, they usually report upon the experience elicited by reporting the presence of the sensation, or rating the sensation (e.g. pain on a visual analogue scale). The assumption is that these reports reflect the capacity of the somatosensory system. However, such examinations do not consist only of somatosensory input. While approaching the body, also visual and possibly auditory information is present. It may well be that the integration of information from several perceptual modalities contributes to the experience of the patient.

This idea of crossmodal interactions has been the subject of extensive research in humans and animals [1-3]. In a typical study of Spence et al. [2], participants were faster and more accurate in making speeded discriminations of tactile targets on the hand when a visual stimulus was presented on the same hand, as opposed to the other hand. Electrophysiological and neuroimaging studies have also confirmed crossmodal links in spatial attention [4-7]. For example, Sambo and Forster [8] recorded somatosensory evoked potentials of increased magnitude when the tactile stimuli applied to one hand were presented concomitantly with a visual cue near that hand. Multisensory interactions have also been proposed for pain, which would facilitate the localization of painful stimuli in close proximity to the body $[9,10]$. De Paepe 
et al. [11] have shown that judgment about the detection of nociceptive stimuli is facilitated by visual stimuli delivered close to the body part on which is applied the nociceptive stimuli.

It seems reasonable to hypothesize that the visual information resulting from an object approaching a body part in close proximity will facilitate the somatosensory processing of that body part. There is some evidence in support of this idea [12], but no study has investigated visuo-tactile interactions in situations resembling clinical and/or QST practices. Therefore, we developed the "In Vivo Approaching Object paradigm", which mimics clinical examinations but also allows for experimental control over stimulus delivery. During each trial, a pen was directed by the experimenter towards a hand of the participant. Once in close proximity to the hand, a vibrotactile stimulus (at sub- or supra-threshold) was delivered to either the approached hand (congruent trials) or the other hand (incongruent trials). The participants' ability to accurately detect and locate the vibrotactile stimulus was measured. In Experiment 1, the pen was directed towards the proximal space of one of the hands. Experiment 2 extended Experiment 1 by also including a condition in which the object was directed towards a location at a further distance from the hands. It was expected that detection accuracy would be higher for congruent than incongruent trials, especially when the pen approached the proximal space of the hand, as opposed to a location at a further distance from it.

\section{EXPERIMENT 1}

\subsection{METHOD}

\subsubsection{Participants}


Thirty undergraduate students took part for course credits (age: $M=21.00 ; \mathrm{SD}=5.59$; range $=17-43$ years; 3 men; 5 left handed). Exclusion criteria were insufficiently corrected visual impairments, the self-report of current medical/psychiatric conditions, or current medication intake affecting somatosensory sensitivity. None of the participants had to be excluded. The study was approved by the Ethics Committee of Ghent University. All participants gave their written informed consent.

\subsubsection{Stimuli and apparatus}

During the In Vivo Approaching Object (IVAO) task, participants were seated with their hands, palms down, resting on a table (see Figure 1). Two square metal plates $\left( \pm 4 \mathrm{~cm}^{2}\right)$ were used as electrical contacts. They were attached to the table, $50 \mathrm{~cm}$ apart from each other and positioned between the thumb and index finger of each hand. The distance between the edge of the table near the participant's trunk - and the plates was $30 \mathrm{~cm}$. At a distance of $55 \mathrm{~cm}$ in front of the edge of the table and $\sim 35 \mathrm{~cm}$ apart from each metal plate, a black fixation cross was presented on the table to prevent participants from shifting their gaze during the task. The participant's head was fixed using a chin wrest. Headphones with continuous white noise (46dB) were used to mask auditory stimuli from the immediate environment. The experimenter was sitting on the other side of the table, at a distance of approximately 1 meter, facing the participant.

\subsubsection{Visual stimuli}

A black pen was held by the experimenter and served as a visual stimulus. The experimenter (LV) held the pen in her left or right hand, and smoothly moved her arm towards one of the two metal plates near the participant's hands, and finally tapped the metal plate. She then moved back to the starting position of the movement. Depending on the plate that had to be 
approached (left or right), the arm closest to that side was used to perform the movement. Tapping the plate triggered the delivery of a tactile stimulus after a time interval of $\sim 2 \mathrm{~ms}$.

\subsubsection{Vibrotactile stimuli}

Two magnet linear actuators (C-2 TACTOR, Engineering Acoustics Inc., Casselberry, Florida) were attached to the sensory territory of the superficial radial nerve of each hand and released vibrotactile stimuli $(50 \mathrm{~ms}$ duration; $50 \mathrm{~Hz})$. The actuators were driven by a selfdeveloped controlling device and software. The intensities of the vibrotactile stimuli were near the perceptual threshold, which was individually determined using an adaptive procedure. The procedure has been used in previous studies $[13,14]$. The procedure consisted of four independent yet randomly intermixed staircases of 20 trials (two series for each hand) randomly administered (80 trials in total). Each series had a starting value of $0.068 \mathrm{Watt}(\mathrm{W})$ for the first stimulus. The intensity decreased each time the participants reported feeling the stimulus, and increased when no sensation was reported. The perceptual threshold was determined for each hand, based upon the mean intensity of the last stimulus of each of the two series of that particular hand. Subthreshold and supra-threshold values were calculated for each hand by respectively subtracting one eighth from the perceptual threshold value, or adding one eighth to it (see [15]).

\subsubsection{Self-report measures}

Participants completed a socio-demographic questionnaire also consisting of the pain grading scale [16], allowing the classification of participants as a function of experienced pain and disability during the last 6 months. Also, current treatment for medical or psychiatric conditions, medication intake and perceived health quality were assessed. Participants also completed the Dutch versions of the Pain Catastrophizing Scale (PCS; [17]) and of the Trait scale 
of the State-Trait Anxiety Inventory (STAI; [18]). The PCS and the STAI were included for a meta-analytic investigation on the role of individual differences in studies on this topic. Individual studies often lack the statistical power to reveal precise estimations of such effects, and hence these data will not further be discussed, but can be requested by addressing the authors.

After each block, a series of self-report items assessed to what extent participants made an effort to fulfill the task; were concentrated on the task; felt tense/fearful during the task; directed their attention towards the pen and the tactile stimuli; experienced the pen as threatening; and used the pen to predict the location of the tactile targets. Each item was rated using a 11-point graphic rating scale $(0=$ "not at all"; $10=$ "very much").

\subsubsection{Procedure}

Participants started with filling out the socio-demographic questionnaire, the PCS and the STAI, after which the staircase procedure followed. Participants were instructed to lay their arms on the table and to find a comfortable position by having the chin wrest and their chair adjusted. A computer screen was placed in front of the participant and instructions about the staircase procedure were given. Following this, the headphones were turned on and the staircase procedure started. First, a visual stimulus (a letter X, 1000ms duration) appeared in the middle of a computer screen, accompanied by a vibrotactile stimulus either on the left or right hand (position unknown to the participant). Participants verbally reported whether they had felt a vibrotactile stimulus ("yes" or "no"). Responses were manually inserted by the experimenter on a keyboard. When the staircase procedure was finished, the computer screen and the headphones were removed. Then, the experimenter calculated the sub- and supra-threshold intensities. 
During the In Vivo Approaching Object (IVAO) task, participants were instructed to keep their hands in a way that each metal plate was positioned between thumb and index finger, and was not being touched (see Figure 1). Participants were also told to fixate the fixation cross during each block. Each trial started by the experimenter approaching the participant's left or right hand with the pen (visual cue), tapping the metal plate next to the hand, and moving the pen back to its original position (near the experimenter's trunk). The experimenter was trained to perform this movement in a standardized manner ( $\sim \mathrm{s}$ approach and $\sim 1 \mathrm{~s}$ retraction). Simultaneously with the tapping, a sub-threshold or supra-threshold vibrotactile stimulus on one or both hands was triggered in $75 \%$ of the trials (target trials). In the remaining $25 \%$ of the trials no stimulus was presented (catch trials). The vibrotactile target could be presented on the same side as the visual cue (congruent unilateral target trials), on the opposite side (incongruent unilateral target trials), or on both sides (bilateral targets trials). Participants verbally responded whether they felt a tactile stimulus, and if so, on which hand (left, right or bilaterally). The four possible responses, i.e. "no sensation", "left sensation", "right sensation", "sensations on both sides", were manually inserted on the keyboard by the experimenter $(0=$ "no sensation"; $4=$ "left sensation"; 6 = "right sensation"; 5 = "sensation on both sides"). Instructions about which hand to approach were visible on a computer screen in front of the experimenter but were masked from the participant's view. The experimenter, however, was blind as to which type of trial (congruent vs. incongruent) was running.

A total of 256 trials was presented, divided across 4 blocks of 64 trials. Each block consisted of 16 catch trials, 16 congruent unilateral trials, 16 incongruent unilateral trials, and 16 bilateral trials. All four types of trials were presented randomly. The majority $(75 \%)$ of the target trials had a stimulus of sub-threshold intensity (i.e. 36 trials), whereas $25 \%$ had a stimulus with 
an intensity slightly above the perceptual threshold (i.e. 12 trials). Supra-threshold targets were presented in order to provide participants a sense of mastery over the task. Catch trials and bilateral trials were added to minimize strategic guessing and to maintain attention to the task. In sum, there were 16 observations ( 4 trials $\times 4$ blocks) per condition for supra-threshold tactile targets and 48 observations (12 trials $\times 4$ blocks) per condition for sub-threshold targets. Participants completed the self-report items after each block.

\subsubsection{Analyses}

Analyses were conducted on Response Accuracy (binomial: correct vs. incorrect) during the unilateral tactile targets. Catch trials and bilateral target trials were discarded. A response was considered as correct when the vibrotactile stimulus was correctly perceived and correctly localized. The independent variables (all within-subject variables) were the Congruency (congruent vs. incongruent) between visual and tactile stimuli, and the Intensity (sub-threshold vs. supra-threshold) of the tactile stimuli.

In order to investigate the effect of Congruency and Intensity upon Response Accuracy, results were analyzed using a linear mixed-effects model with a logit link function, as implemented in the R package lme4 [19]. Mixed effects models account for the correlations in within-subjects data by estimating subject-specific deviations (or random effects) from each population-level factor (or fixed factor) of interest (see [20] for an elaboration).

The analysis consisted of three steps. First, all relevant factors and interactions were entered in the model as fixed factors, and we assessed whether it was necessary to add a random effect for each of the fixed factors in the analysis: if a random effect significantly increased the fit of the model, it was included in the final model (see Supplementary File 1, illustrating the 
building of the full model). By default, a random effect was added introducing adjustments to the intercept of the Subject variable. In the second step, we searched for the most parsimonious model that fitted the data. To achieve this, the full model was systematically restricted, comparing the goodness of fit using likelihood ratio tests and Akaike's information criterion [21] (see Supplementary File 2, showing the restricting of the full model). As we were interested in all included variables, fixed effects were never removed from the model. Finally, in the third step, we inspected the ANOVA table of the final model, and tested specific hypotheses about possible main effects or interactions (for a similar approach see [22-24]) (see Supplementary File 3, showing the ANOVA table of the final model).

\subsection{RESULTS}

\subsubsection{Staircase}

The mean value for the perceptual threshold was significantly different between the left hand $(M=0.038 \mathrm{~W}, \mathrm{SD}=0.021)$, and the right hand $(M=0.021 \mathrm{~W}, \mathrm{SD}=0.011, \mathrm{t}(29)=4.02, p$ $<0.001)$. This effect was not different between individuals with right hand dominance $(\mathrm{n}=25)$ and individuals with left hand dominance $(\mathrm{n}=5, \mathrm{t}(28)=-1.37, p=0.18)$, albeit the low number of individuals with left hand dominance may have led to a reduced statistical power.

\subsubsection{Self-report measures}

Participants reported to be highly concentrated $(M=7.49$; SD $=1.35)$ and to have put much effort to the task $(M=8.09 ; \mathrm{SD}=1.11)$. Also, participants reported not to be tense/fearful during the task $(M=1.49 ; \mathrm{SD}=1.62)$. The self-reported attention directed towards the tactile targets was high $(M=8.61, \mathrm{SD}=0.87)$, whereas attention towards the pen was rather low $(M=$ $2.78, \mathrm{SD}=1.90$ ). In addition, participants reported not having used the position of the pen to 
predict the tactile target $(M=1.03, \mathrm{SD}=1.48)$, nor was it experienced as being threatening $(M=$ $0.90, \mathrm{SD}=1.27)$

\subsubsection{Response accuracy to vibrotactile stimuli}

The model that demonstrated the best fit included only the main effects of the fixed factors, a random subject-based intercept, and a random effect both for Intensity and Congruency.

There was a significant main effect of Intensity $\left(\chi^{2}(1)=108.38, p<0.001, \beta=-1.57,95 \%\right.$ CI [1.86 to -1.27$])$, meaning that Response Accuracy was higher for supra-threshold targets trials $(M$ $=87.40 \% ; \mathrm{SD}=12.19)$ compared to sub-threshold targets trials $(M=64.38 \% ; \mathrm{SD}=17.18)$. In addition, there was a significant main effect of Congruency $\left(\chi^{2}(1)=17.85, p<0.001, \beta=-0.65\right.$, 95\% CI [-0.96 to -0.35$])$ revealing that Response Accuracy was higher in congruent $(M=$ 75.94\%; $\mathrm{SD}=13.58)$ trials, compared to incongruent $(M=64.32 \%$; $\mathrm{SD}=19.75)$ target trials (Figure 2, top panel).

\subsection{DISCUSSION}

Experiment 1 shows that Response Accuracy, i.e. the ability to perceive and correctly localize the vibrotactile stimuli, was higher when the target location of the approaching visual cue was congruent with the tactile stimulation, as opposed to when it was incongruent. In other words, tactile processing was facilitated at the hand that was approached by the pen. Because in Experiment 1 all visual cues were presented in close proximity to the hands, it was not possible to determine whether the visuo-tactile spatial congruency effect resulted from a crossmodal processing facilitation due to the visual object approaching the proximal location of the stimulated limb, or whether it merely resulted from a response priming effect (i.e. cueing the left 
vs. right hemi-space primes a response related to that particular hemi-space; see Spence and Driver 1997 for comments on this issue). Therefore, in Experiment 2, the distance of the visual cues towards the hands was manipulated, resulting in an approaching movement close to the participant's hand (i.e. peripersonal space) or far from it (i.e. extrapersonal space).

\section{EXPERIMENT 2}

\subsection{METHOD}

\subsubsection{Participants}

Thirty-five undergraduate students took part for course credits (age: $\mathrm{M}=19.66, \mathrm{SD}=$ 4.80 , range $=17-44$ years; 12 men; 9 left handed). Inclusion and exclusion criteria were the same as in Experiment 1. Due to apparatus failure, data storage was incomplete for 12 participants. As a result, 23 participants (age: $\mathrm{M}=19.04, \mathrm{SD}=2.53$, range $=17-27$ years; 6 men; 7 left handed) were included for further analysis. The study was approved by the Ethics Committee of Ghent University. All participants gave their written informed consent.

\subsubsection{Stimuli and apparatus}

Stimuli, apparatus, set-up and procedure were similar as in Experiment 1. The main difference was that four - instead of two - metal plates were attached to the table. Two plates were positioned between the thumb and index finger (close plates). Two additional plates were placed further away in front of the participants, at $50 \mathrm{~cm}$ from the close plates on the same sagittal line (far plates) (see Figure 3).

\subsubsection{Visual stimuli}


The same pen was held by the experimenter as a visual stimulus. Now, the pen could approach four different locations defined by respective positions of the two close and the two far contact plates.

\subsubsection{Vibrotactile stimuli}

The parameters of the vibrotactile stimuli were the same as in Experiment 1, including the staircase procedure to select stimulus intensity.

\subsubsection{Self-report measures.}

The questionnaires and self-report measures were identical to those in Experiment 1.

\subsubsection{Procedure}

The procedure for the self-report measures and the staircase of Experiment 2 were the same as in Experiment 1. The IVAO task was also identical for the stimulation blocks during which the close plates were approached and contacted by the pen. During the other blocks with the far plates, the experimenter was sitting $50 \mathrm{~cm}$ further away from the participants in order to maintain the same distance for the approaching movement. The experimenter was also trained to keep about the same speed of movement between the two types of blocks.

In this experiment, 384 trials, divided into six blocks of 64 trials, were presented. Which plate was to be approached and touched (close vs. far) alternated between blocks. The order of the blocks was randomly assigned. In each block, there were 16 catch trials, 16 congruent unilateral target trials, 16 incongruent unilateral target trials and 16 bilateral target trials (randomly presented). The proportion of $25 \%$ of the stimuli at supra-threshold intensity and $75 \%$ at sub-threshold intensity was identical as in Experiment 1, resulting in 12 supra-threshold and 36 
sub-threshold trials. The number of observations per condition was 12 (4 trials $\mathrm{x} 3$ identical blocks) for supra-threshold targets, and 36 (12 trials x 3 identical blocks) for sub-threshold targets.

\subsubsection{Analyses}

Similar analyses as in Experiment 1 were performed. Response Accuracy was analyzed using a linear mixed-effects model with Congruency (congruent vs. incongruent), Cue Distance (close vs. far) and Intensity (low vs. high) as independent within-subjects variables. Follow-up analyses were used when appropriate (see Supplementary Files 4-6, illustrating the model building procedure).

\subsection{RESULTS}

\subsubsection{Staircase}

Perceptual thresholds did not differ between the left and the right hands (left: $M=0.030$, $\mathrm{SD}=0.022 ;$ right: $M=0.035, \mathrm{SD}=0.023 ; \mathrm{t}(22)=-0.66, p=0.52)$. Also, there were no differences in perceptual threshold between individuals with right hand dominance $(n=16)$ and individuals with left hand dominance $(\mathrm{n}=7, \mathrm{t}(21)=1.05, p=0.31)$.

\subsubsection{Self-report measures}

Results from the self-report measures were similar to Experiment 1 . The amount of effort $(M=7.99, \mathrm{SD}=1.34)$ and concentration $(M=7.61, \mathrm{SD}=1.18)$ during the task was high. Mean self-reported fear/tension was low $(M=1.49, \mathrm{SD}=1.56)$. Furthermore, the amount of attention directed towards the tactile stimuli was high $(M=8.44, \mathrm{SD}=1.06)$, whereas attention towards the pen was quite low $(M=2.99, \mathrm{SD}=1.98)$. Participants also reported not having used the 
position of the pen to the predict the location of the tactile stimuli $(M=1.40, \mathrm{SD}=1.45)$ and felt not threatened by it $(M=0.97, \mathrm{SD}=1.43)$.

\subsubsection{Response Accuracy for vibrotactile stimuli}

The model that demonstrated the best fit included the main effects of the fixed factors, an interaction between Congruency and Distance, a random subject-based intercept, and a random effect for Intensity, Congruency and Distance.

We found a significant main effect of Congruency $\left(\chi^{2}(1)=27.45, p<0.001, \beta=-0.75\right.$, 95\% CI [-1.02 to -0.47$])$, indicating that Response Accuracy was higher for congruent trials $(M=$ $71.24 \%, \mathrm{SD}=13.04)$ than for incongruent trials $(M=62.73 \%, \mathrm{SD}=18.27)$. A significant main effect of Distance $\left(\chi^{2}(1)=26.42, p<0.001, \beta=-0.66,95 \%\right.$ CI $[-0.91$ to -0.41$\left.]\right)$ indicated a higher Response Accuracy when the approaching cue was close to the hands $(\mathrm{M}=70.33 \%, \mathrm{SD}=$ 15.45), compared to when the approaching cue was far from the hands $(\mathrm{M}=63.63 \%, \mathrm{SD}=$ 15.61). Response Accuracy was also higher for supra-threshold target trials $(\mathrm{M}=84.15 \%, \mathrm{SD}=$ 14.03) than for sub-threshold target trials $(\mathrm{M}=61.26 \%, \mathrm{SD}=16.41)$ as shown by a main effect of Intensity $\left(\chi^{2}(1)=76.61, p<0.001, \beta=-1.47,95 \%\right.$ CI $[-1.80$ to -1.14$\left.]\right)$. Finally, there was a significant interaction between Congruency and Distance $\left(\chi^{2}=16.10, p<0.001, \beta=0.57,95 \%\right.$ CI [0.29 to 0.85]). Follow-up tests indicate that the difference in Response Accuracy between congruent and incongruent trials was significant when cues were presented nearby $\left(\chi^{2}(1)=27.45\right.$, $p<0.001$ ), but not when they were presented far $\left(\chi^{2}(1)=1.63, p=0.20\right.$ ) (Figure 2, bottom panel).

\subsection{DISCUSSION}


In Experiment 2, the visuo-tactile congruency effect from Experiment 1 was replicated: Response Accuracy was higher when the visual and tactile stimuli were presented on the same location (congruent), compared to the opposite location (incongruent). Moreover, we found that this visuo-tactile spatial interaction was only significant when visual cues were presented near as opposed to far from the stimulated hands.

\section{GENERAL DISCUSSION}

This study investigated whether viewing someone approaching your body alters the perception of a co-occurring tactile stimulus. It was found that the detection accuracy of nearthreshold vibrotactile targets on the hands was higher for the visually cued (i.e. approached) hand as compared to the opposite hand (Experiment 1). Moreover, Experiment 2 revealed that this visuo-tactile spatial congruency effect was only present when the pen approached the hand in close proximity (peripersonal space). It was not present when the pen was further away from the hands.

These results are in line with several studies demonstrating the influence of crossmodal interaction on the processing of somatosensory stimuli $[2,25]$. However, in most of those studies static - as opposed to dynamic - visual stimuli have been used, reducing the generalizability to real-life (clinical) situations. Yet, since an important function lies within localizing stimuli events surrounding the body, it seems reasonable that stimuli approaching the body require full attentional processing. Therefore, this study has investigated and confirmed the enhancing effect of approaching (i.e. dynamic) visual stimuli on tactile sensitivity. The latter might especially be important for health care providers, performing somatosensory examinations on patients by 
approaching them with a measuring device or with their hands. For example, during the examination of neuropathic pain, quantitative sensory testing (QST) is a well-used diagnostic tool that requires approaching a patient while measuring sensory symptoms. Also, when doctors verify the diagnosis of complex regional pain syndrome, they need to assess a series of (sensory) symptoms (e.g. hyperesthesia) by approaching and touching the affected hand (e.g. with a von Frey filament or algometer) [26,27]. In these cases, approaching the patient might lead to a momentary increased sensitivity for touch, and thereby to an overestimation of the evaluated symptom. Based on this study, it is not yet possible to determine the magnitude of this increased sensitivity nor to conclude that it could effectively lead to misdiagnosis. However, it may be useful for clinicians to be aware of this phenomenon and to take it into account when conducting somatosensory evaluations on patients. For example, doctors could choose to instruct patients to close their eyes while being examined, to prevent visual feedback [28].

During the last decades, researchers have gained interest in the interaction between visual and somatosensory information near the body. Several authors have proposed that when encountering a stimulus event surrounding the body, combining information from the different senses (i.e. crossmodal interactions) might provide the best estimate of the external event $[3,25,29]$. Researchers have conducted extensive behavioral, as well as electrophysiological and brain imaging research to support this notion [3-7]. There it was also found that these crossmodal influences mainly take place near the body [30,31], in the so-called peripersonal space [32]. Kandula, Hofman and Dijkerman [33] explain that information coming from peripersonal space can be of higher significance in terms of processing as: $i$ ) this region is the only space surrounding the body in which stimuli can be interacted with; ii) stimuli in this region (close to the body) could be potentially more harmful for the body's integrity. Our results corroborate 
these findings. Which neural/psychological mechanisms underlie these findings is still subject of investigation. There are at least three possible explanations (see review in [34]).

First, our findings may be explained by spatial attention, meaning that the perception of a stimulus in one modality will attract attention towards its location, increasing the chance of nearby stimuli from other modalities being detected [34]. Apart from this rather bottom-up approach to spatial attention, a top-down anticipatory component might also increase attention towards the approached body part. A recent study [33] has suggested that a prediction mechanism underlies the effect of approaching visual stimuli on temporal/spatial tactile judgments. Accordingly, participants in our study could have been hard-wired to anticipate the occurrence of a tactile stimulus on their approached hand, even if this was only the case in a minority of the trials (25\% congruent unilateral target trials and $25 \%$ bilateral target trials). This top-down anticipation may then have evoked heightened spatial attention to the location of the approached body part, resulting in higher detection accuracy.

Second, stimulus-driven 'multisensory integration' may as well lay at the foundation of crossmodal interaction effects. This implies that information from different sensory modalities is processed in unity, as if it were originating from a common source of input, provided that these multiple sources of input correspond in both time and space [34].

A third and related explanation originates from animal studies demonstrating visuo-tactile integration near the body at the single-neuron level [12,35-38]. Neurons in brain areas such as the ventral premotor area and the ventral intraparietal sulcus have been shown to process inputs from different sensory modalities [12,37-39]. More specifically, neurons in this region are found to have multimodal receptive fields (RFs), meaning that they respond to stimuli from different 
modalities who are present within a common region of space on and/or around the body. Graziano et al. [39], for example, have demonstrated that bimodal neurons from the ventral premotor cortex in monkeys fire for both tactile and visual stimuli when visual stimuli are in proximity to the tactile RF. Especially visual stimuli approaching the body were found to be targeted by these bimodal neurons [12,39]. One of the key features of these neurons is that their visual RF is spatially locked to the tactile RFs, meaning that they move in space with the body part the code, independently of the position of the triggering visual stimuli on the retina. This functional property of bimodal neurons might explain why participants in our study were better in detecting tactile targets who were accompanied by a visual cue in the peri-hand space (congruent-close unilateral target trials) as compared to the contralateral hemi-space (incongruent-close unilateral target trials) and the extrapersonal space (far unilateral target trials). Additional research is needed to determine which of these underlying mechanisms is responsible for the increased tactile sensitivity after visual approach.

There are some limitations to this study. First, the approaching movement was not mechanically standardized. Therefore, the exact duration and trajectory of the stimulus could have slightly differed between trials. Second, the use of an ecologically valid stimulus such as an approaching hand has some disadvantages. Although our studies show that approaching someone with real hands has particular effects, we have less control over potentially confounding effects, such as, for example, the increasing size of an approaching object on the retina. However, despite the fact the retinal size of visual stimuli are usually controlled in experimental settings, this effect is unlikely to have played a major role in our date since it was shown there is no strict scaling relationship between retinal image size and the importance of its perception. For instance Murray et al. [40] have shown that the V1 cortical responses to visual stimuli do not merely depend of 
their retinal sizes but already integrate other contractual information such as the perception of deepness. Third, there was a lack of orthogonality between the direction of the visual cue (left vs. right) and the direction of the responses (also left vs. right). Non-orthogonal response mapping can lead to the misconception that actual crossmodal interactions are at work, whereas it might only be hemispheric activation, priming a congruent response [34]. However, the lack of crossmodal interactions in extrapersonal space in Experiment 2 proves that response priming cannot have (fully) explained the current results. Fourth, the detection and localization of tactile stimuli was measured as outcome variable, but not its rated intensity, impeding us to draw any conclusions on the size of changes in tactile sensitivity. Related to that, participants in our study did not experience pain nor did they undergo painful target stimuli, although this might often be the case in clinical examinations. The IVAO paradigm may be easily adapted to address these pertinent questions. Future research should especially meet the need for multisensory research in the context of pain. Despite the high current popularity of this topic, clear evidence is still lacking $[41,42]$.

In conclusion, the current study provides evidence on the effect of nearby approaching movements on tactile detection accuracy. We developed the In Vivo Approaching Object paradigm as a straightforward and ecologically valid method to measure visuo-tactile interactions around the body. Our findings suggest that changes in tactile sensitivity due to approaching movements might not only occur in research settings, but also in medical settings. 


\section{Acknowledgments}

We express our gratitude to Delia Mensitieri who contributed to the data collection of this study. This study is part of a research project (G.0058.11N) granted by the Research Foundation -

Flanders, Belgium (Fonds Wetenschappelijk Onderzoek [FWO]). A.L. De Paepe is a PhD fellow of the Research Foundation - Flanders (FWO). Valéry Legrain is Research Associate at the Fund for Scientific Research of the French speaking Community of Belgium (F.R.S.-FNRS).

\section{Conflict of interest statement}

The authors have no conflict of interested related to the present article. 


\section{REFERENCES}

[1] Spence C, Driver J. Audiovisual links in exogenous covert spatial orienting. Percept Psychophys 1997;59:1-22. doi:10.3758/BF03206843.

[2] Spence C, Nicholls ME, Gillespie N, Driver J. Cross-modal links in exogenous covert spatial orienting between touch, audition, and vision. Percept Psychophys 1998;60:544-57. doi:10.3758/BF03206045.

[3] Driver J, Spence C. Attention and the crossmodal construction of space. Trends Cogn Sci 1998;2:254-62. doi:10.1016/S1364-6613(98)01188-7.

[4] Macaluso E, Driver J. Spatial attention and crossmodal interactions between vision and touch. Neuropsychologia 2001;39:1304-16. doi:10.1016/S0028-3932(01)00119-1.

[5] Calvert GA, Campbell R, Brammer MJ. Evidence from functional magnetic resonance imaging of crossmodal binding in the human heteromodal cortex. Curr Biol 2000;10:64957. doi:10.1016/S0960-9822(00)00513-3.

[6] Sathian K, Zangaladze A, Hoffman JM, Grafton ST. Feeling with the mind's eye. vol. 8. 1997. doi:10.1097/00001756-199712220-00008.

[7] Calvert GA, Brammer MJ, Bullmore ET, Campbell R, Iversen SD, David AS. Response amplification in sensory-specific cortices during crossmodal binding. vol. 10. 1999. doi:10.1097/00001756-199908200-00033.

[8] Sambo CF, Forster B. An ERP investigation on visuotactile interactions in peripersonal and extrapersonal space: evidence for the spatial rule. J Cogn Neurosci 2009;21:1550-9. doi:10.1162/jocn.2009.21109.

[9] Haggard P, Iannetti GD, Longo MR. Spatial sensory organization and body representation in pain perception. Curr Biol 2013;23. doi:10.1016/j.cub.2013.01.047.

[10] Legrain V, Torta DM. Cognitive psychology and neuropsychology of nociception and pain. In: Pickering G, Gibson S, editors. Pain, Emot. Cogn. a Complex Nexus, Springer; 2015, p. 3-20.

[11] De Paepe AL, Crombez G, Spence C, Legrain V. Mapping nociceptive stimuli in a peripersonal frame of reference: Evidence from a temporal order judgment task. Neuropsychologia 2014;56:219-28. doi:10.1016/j.neuropsychologia.2014.01.016.

[12] Graziano MSA, Gross CG. The Representation of Extrapersonal Space: A Possible Role for Bimodal Visual-Tactile Neurons. Cogn. Neurosci., 1995, p. 1021-34. 
[13] Vandenbroucke S, Crombez G, Harrar V, Brusselmans G, Devulder J, Spence C, et al. Fibromyalgia patients and controls are equally accurate in detecting tactile stimuli while observing another in pain : an experimental study 2014:2548-59. doi:10.3758/s13414-0140729-9.

[14] Vandenbroucke S, Crombez G, Loeys T, Goubert L. Observing another in pain facilitates vicarious experiences and modulates somatosensory experiences. Front Hum Neurosci 2014;8:1-14. doi:10.3389/fnhum.2014.00631.

[15] Press C, Taylor-Clarke M, Kennett S, Haggard P. Visual enhancement of touch in spatial body representation. Exp Brain Res 2004;154:238-45. doi:10.1007/s00221-003-1651-x.

[16] Von Korff M, Ormel J, Keefe FJ, Dworkin SF. Grading the severity of chronic pain. Pain 1992;50:133-49. doi:10.1016/0304-3959(92)90154-4.

[17] Sullivan MJL, Bishop SR, Pivik J. The Pain Catastrophizing Scale: Development and validation. Psychol Assess 1995;7:524-32. doi:10.1037/1040-3590.7.4.524.

[18] Spielberger CD. State-Trait Anxiety Inventory. Anxiety 1987;19:2009.

[19] Pinheiro JC, Bates DM. Mixed Effects Models in Sand S-PLUS. New York SpringerVerlag 2000. doi:10.1007/978-1-4419-0318-1.

[20] West BT, Welch KB, Ga AT, Crc H. Linear mixed models. A Practical Guide Using Statistical Software. vol. 27. 2007. doi:10.1002/sim.3167.

[21] Hu S. Akaike information criterion. Cent Res Sci Comput 2007. doi:10.2307/1268842.

[22] De Ruddere L, Goubert L, Prkachin KM, Louis Stevens MA, Van Ryckeghem DML, Crombez G. When you dislike patients, pain is taken less seriously. Pain 2011;152:23427. doi:10.1016/j.pain.2011.06.028.

[23] De Ruddere L, Goubert L, Stevens M, Amanda AC, Crombez G. Discounting pain in the absence of medical evidence is explained by negative evaluation of the patient. Pain 2013;154:669-76. doi:10.1016/j.pain.2012.12.018.

[24] Verbruggen F, Aron AR, Stevens MA, Chambers CD. Theta burst stimulation dissociates attention and action updating in human inferior frontal cortex. Proc Natl Acad Sci U S A 2010;107:13966-71. doi:10.1073/pnas.1001957107.

[25] Làdavas E, Farnè A. Visuo-tactile representation of near-the-body space. J Physiol Paris 2004;98:161-70. doi:10.1016/j.jphysparis.2004.03.007.

[26] Harden RN, Bruehl S, Perez RSGM, Birklein F, Marinus J, Maihofner C, et al. Validation of proposed diagnostic criteria (the "budapest Criteria") for Complex Regional Pain Syndrome. Pain 2010;150:268-74. doi:10.1016/j.pain.2010.04.030. 
[27] Harden RN. Objectification of the diagnostic criteria for CRPS. Pain Med 2010;11:12125. doi:10.1111/j.1526-4637.2010.00909.x.

[28] Keizer D, van Wijhe M, Post WJ, Wierda JMKH. Quantifying allodynia in patients suffering from unilateral neuropathic pain using von frey monofilaments. Clin J Pain 2007;23:85-90. doi:10.1097/01.ajp.0000210950.01503.72.

[29] Driver J, Spence C. Cross-modal links in spatial attention. Philos Trans R Soc Lond B Biol Sci 1998;353:1319-31. doi:10.1098/rstb.1998.0286.

[30] Làdavas E, di Pellegrino G, Farnè a, Zeloni G. Neuropsychological evidence of an integrated visuotactile representation of peripersonal space in humans. J Cogn Neurosci 1998;10:581-9. doi:10.1162/089892998562988.

[31] Ladavas. Functional and dynamic properties of visual perispace. Trends Cogn Sci Sci 2004;6:17-22.

[32] Rizzolatti G, Fadiga L, Fogassi L, Gallese V. The space around us. Science 1997;277:1901. doi:10.1126/science.277.5323.190.

[33] Kandula M, Hofman D, Chris Dijkerman H. Visuo-tactile interactions are dependent on the predictive value of the visual stimulus. Neuropsychologia 2014:1-9. doi:10.1016/j.neuropsychologia.2014.12.008.

[34] Spence C, Driver J. Crossmodal space and crossmodal attention. 1st ed. New York: Oxford University Press; 2004.

[35] Rizzolatti G, Luppino G, Matelli M. The organization of the cortical motor system: New concepts. Electroencephalogr Clin Neurophysiol 1998;106:283-96. doi:10.1016/S00134694(98)00022-4.

[36] Rizzolatti G, Scandolara C, Matelli M, Gentilucci M. Afferent properties of periarcuate neurons in macaque monkeys. II. Visual responses. Behav Brain Res 1981;2:147-63. doi:10.1016/0166-4328(81)90053-X.

[37] Graziano MSA, Gross CG. Visual responses with and without fixation: Neurons in premotor cortex encode spatial locations independently of eye position. Exp Brain Res 1998;118:373-80. doi:10.1007/s002210050291.

[38] Duhamel JR, Colby CL, Goldberg ME. Ventral intraparietal area of the macaque: congruent visual and somatic response properties. J Neurophysiol 1998;79:126-36.

[39] Graziano MSA, Hu XT, Gross CG. Visuospatial properties of ventral premotor cortex. J Neurophysiol 1997;77:2268-92. 
[40] Murray SO, Boyaci H, Kersten D. The representation of perceived angular size in human primary visual cortex. Nat Neurosci 2006;9:429-34. doi:10.1038/nn1641.

[41] Valentini E, Kock K, Aglioti SM. Seeing one's own painful hand positioned in the contralateral space reduces subjective reports of pain and modulates laser evoked potentials. J Pain 2015.

[42] Torta DM, Legrain V, Mouraux A. Looking at the hand modulates the brain responses to nociceptive and non-nociceptive somatosensory stimuli but does not necessarily modulate their perception. Psychophysiology 2015;in press. 
Figures
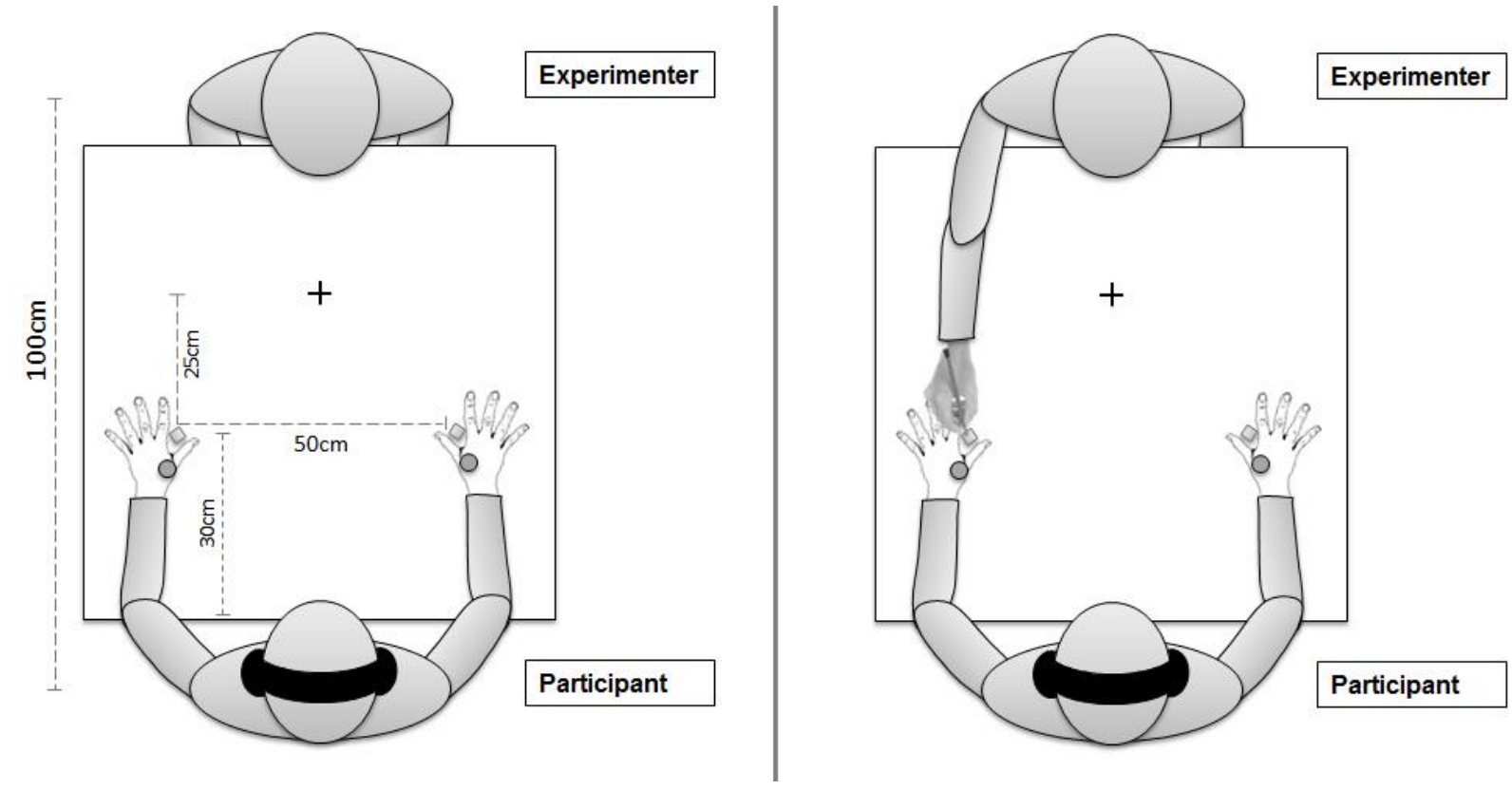

Figure 1. Experimental set-up of the In Vivo Approaching Object task in Experiment 1.

Left panel: Participants are seated across the experimenter with a close contact plate between either thumb and index finger. Right panel: during each trial, the experimenter approaches one of the participant's hands with the pen, taps the contact plate and returns to the starting position (see left panel). 


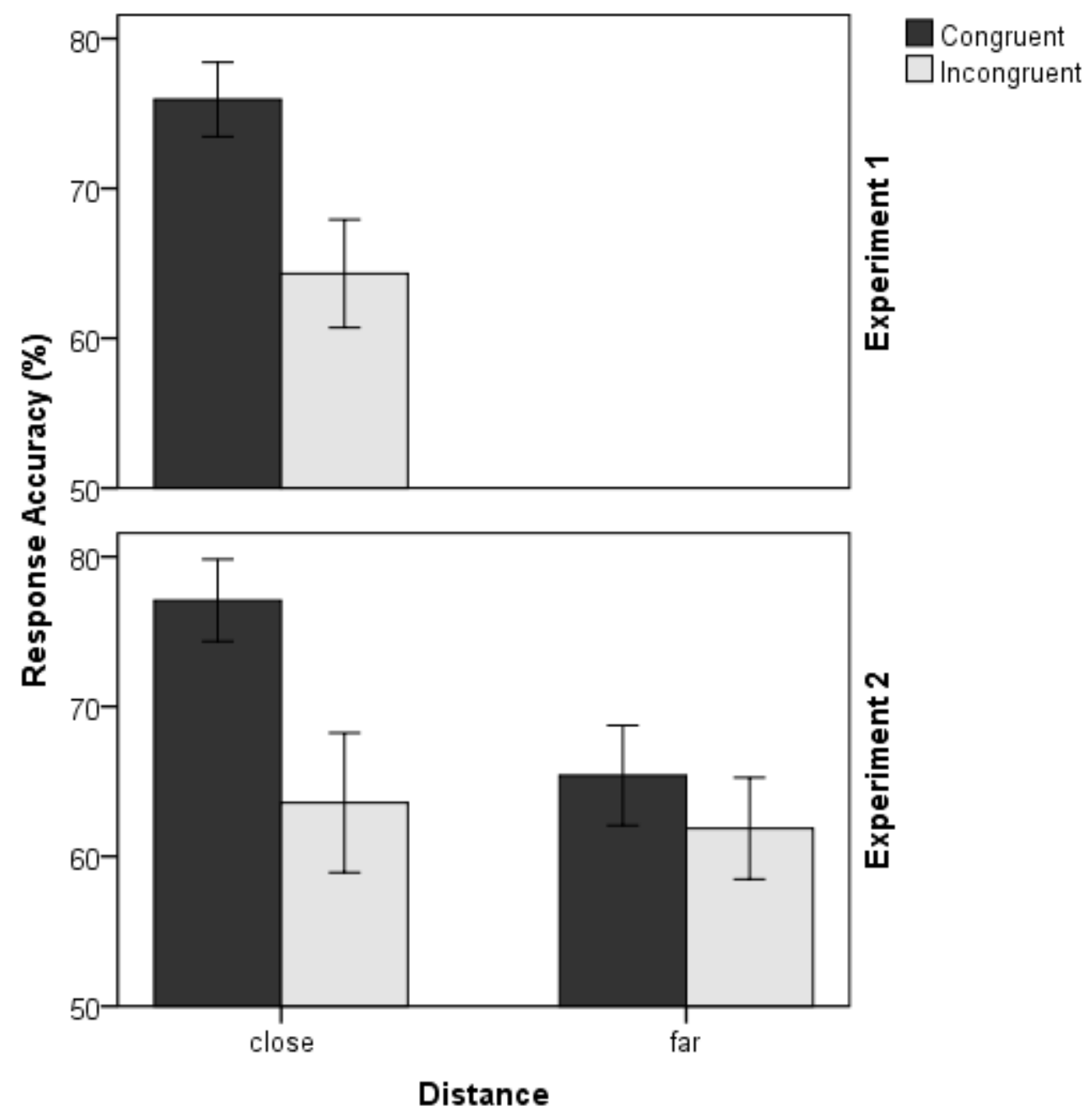

Figure 2. Response Accuracy (\%) in Experiments 1 and 2, depending on Congruency and Distance of the visual cues. Error bars represent standard error of the mean (SEM) 

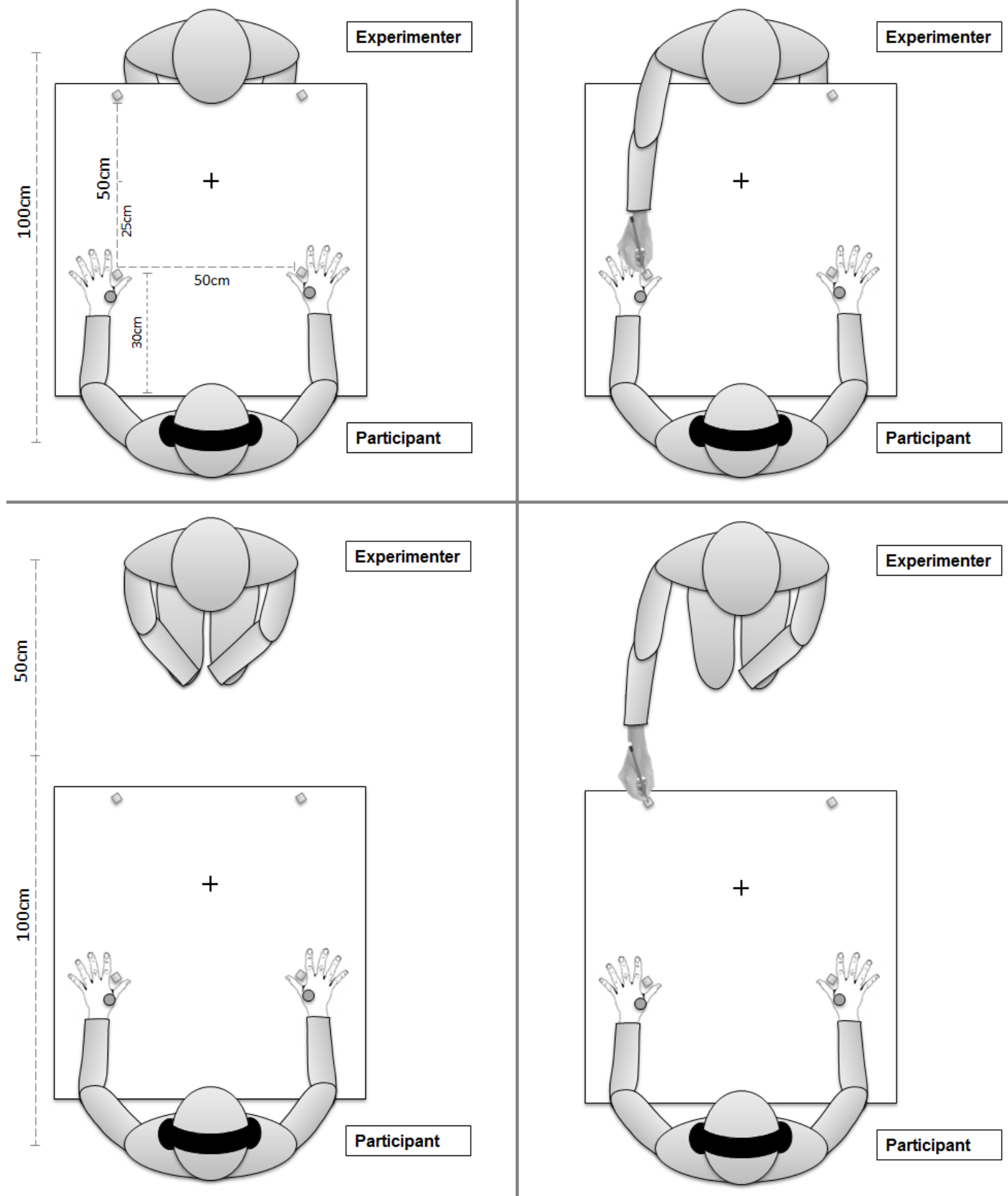

Figure 3. Experimental set-up of In Vivo Approaching Object IVAO task in Experiment 2.

Top panels: close condition as seen in Experiment 1. Bottom left panels: far condition. The experimenter is seated at a further distance from the participant (left bottom panel), allowing a 
similar approaching movement as in the close condition, but now towards the two far contact plates (right bottom panel). 